\title{
Placental mitochondrial adaptations in preeclampsia associated with progression to term delivery
}

\author{
Olivia J. Holland (10 ', James S. M. Cuffe ${ }^{1,2}$, Marloes Dekker Nitert $\mathbb{B}^{3,4}$, Leonie Callaway ${ }^{3,5}$, Keith A. Kwan Cheung ${ }^{1}$,
} Filip Radenkovic ${ }^{1}$ and Anthony V. Perkins ${ }^{1}$

\begin{abstract}
Preeclampsia is a devastating pregnancy disorder. Severity varies widely, and while severe preeclampsia often requires pre-term delivery, women with mild preeclampsia may reach term with minor interventions. The mechanisms that mediate disease severity are poorly understood, but may include adaptive processes by the placenta. We aimed to establish whether in pregnancies that reached term and those that delivered pre-term, the placental response to preeclampsia was intrinsically different, and explore potential adaptive mechanisms. Hydrogen peroxide production and antioxidant activity were increased in term preeclamptic placentae, whereas pre-term preeclamptic placentae had reduced hydrogen peroxide production and reduced function of the antioxidant system superoxide dismutase compared to control placentae. Markers of mitochondrial fission/fusion, apoptosis and the expression level of mitochondrial complexes were differentially disrupted in term compared to pre-term preeclamptic placentae. Mitochondrial respiration and content were increased in term preeclamptic placentae, but mitochondria had a lower respiratory reserve capacity. Mitochondrial respiration and hydrogen peroxide production were increased in healthy term placentae after in vitro hypoxia/reoxygenation. Placentae from preeclamptic pregnancies that reached term showed multiple adaptions that were not present in pre-term preeclamptic placentae. Increased antioxidant activity, and expression of markers of mitochondrial fusion and apoptotic suppression, may relate to salvaging damaged mitochondria. Increased mitochondrial respiration may allow ongoing tissue function even with reduced respiratory efficiency in term preeclamptic pregnancies. Response after in vitro hypoxia/reoxygenation suggests that disruption of oxygen supply is key to placental mitochondrial adaptations. Reactive oxygen species signalling in term preeclamptic placentae may be at a level to trigger compensatory antioxidant and mitochondrial responses, allowing tissue level maintenance of function when there is organelle level dysfunction.
\end{abstract}

\section{Introduction}

Preeclampsia affects $1-5 \%$ of pregnancies and is estimated to be responsible for 70,000-80,000 maternal deaths and 500,000 perinatal deaths worldwide every year $^{1,2}$. Preeclampsia has very limited treatment options

\footnotetext{
Correspondence: Olivia J. Holland (o.holland@griffith.edu.au)

${ }^{1}$ School of Medical Science, Griffith University, Gold Coast Campus, Southport, QLD, Australia

${ }^{2}$ School of Biomedical Sciences, The University of Queensland, St Lucia, QLD, Australia

Full list of author information is available at the end of the article.

Edited by C. Munoz-Pinedo
}

and can only be cured by delivery. The disorder can therefore require pre-term delivery, which itself is associated with poor offspring health outcomes ${ }^{2,3}$.

Preeclampsia is a multi-system disorder characterised by new onset maternal hypertension and endothelial dysfunction (commonly diagnosed through proteinuria). This broad symptomatic definition likely represents a disorder with complex aetiology. Indeed, sub-types of preeclampsia are recognised in the research literature ${ }^{4}$ and clinical management guidelines ${ }^{5}$. The symptoms of preeclampsia can rapidly become severe enough to result

\section{(c) The Author(s) 2018}

(c) (i) Open Access This article is licensed under a Creative Commons Attribution 4.0 International License, which permits use, sharing, adaptation, distribution and reproduction cc) in any medium or format, as long as you give appropriate credit to the original author(s) and the source, provide a link to the Creative Commons license, and indicate if changes were made. The images or other third party material in this article are included in the article's Creative Commons license, unless indicated otherwise in a credit line to the material. If material is not included in the article's Creative Commons license and your intended use is not permitted by statutory regulation or exceeds the permitted use, you will need to obtain permission directly from the copyright holder. To view a copy of this license, visit http://creativecommons.org/licenses/by/4.0/. 
in maternal mortality (e.g., through organ failure and seizures), thus necessitating pre-term delivery as an intervention. Alternatively, the disorder can remain mild enough to allow pregnancy progression to term delivery. The different features of preeclampsia sub-types may represent varied aetiologies or the activation of different damage/repair pathways; however, these differences have only been characterised to a limited degree.

The placenta is central to the pathogenesis of preeclampsia. The precise pathophysiological mechanisms are not fully understood, but there is substantial evidence that early defects in placentation result in perturbed maternal blood flow to the placenta as pregnancy progresses, leading to further placental damage and oxidative stress $^{6,7}$. Disrupted delivery of blood, and therefore oxygen, to a wide range of tissues is known to cause damage to the mitochondrial electron transfer system (ETS), which leads to increased production of reactive oxygen species $(\mathrm{ROS})^{8}$. Antioxidant systems (e.g., superoxide dismutase, catalase, glutathione peroxidase), as well as coordinated mitochondrial (e.g., biogenesis, fission/ fusion of mitochondria network) and cellular (e.g., autophagy, apoptosis) adaptations constitute the cellular response to ROS and ROS-induced damage ${ }^{9,10}$. The relative levels of these responses versus ROS levels determine the effectiveness at counteracting damage. Mitochondrial damage and ROS production have been well characterised in the heart following myocardial infarction, and the ability of cardiac tissue to respond to ischaemia then to reperfusion is critical to outcomes ${ }^{11-13}$. Furthermore, the dynamic response of mitochondria can lead to adaptive changes in tissues to better suit cellular metabolism to the environment. For example, in skeletal muscle, exercise enhances mitochondrial respiration and biosynthesis ${ }^{14,15}$, and in cardiac muscle, exposure to low levels of ROS before a major insult results in a range of adaptions that lead to better outcomes (termed ischaemic preconditioning) ${ }^{16-18}$.

There is evidence that severe preeclampsia is associated with increased ROS and reduced placental antioxidant function $^{19,20}$, and in vitro antioxidant treatment can improve trophoblast responses to oxidative stress ${ }^{21-23}$. However, clinical trials of antioxidants for the treatment of preeclampsia have been generally unsuccessful ${ }^{24,25}$, and ROS-mediated signalling may be important for maintaining placental function ${ }^{26}$.

To investigate if the less severe forms of preeclampsia represent successful countering of adverse conditions, the current study compares antioxidant function and mitochondrial dynamics between placentae from control and preeclamptic pregnancies that either reached term or were delivered pre-term. The direct effect of hypoxia/ reoxygenation on placental mitochondria is also investigated in healthy placental tissue.

\section{Materials and methods}

\section{Materials, tissue collection, and ethics}

Chemicals were purchased from Sigma Aldrich, Australia unless noted otherwise. This study was approved by the Queensland Government Human Research Ethics Committee, Australia (HREC/08/QRBW/1 and HREC/ 14/QPCH/246), Griffith University (MSC/05/15/HREC), and the University of Queensland (UQ 2009000115). All tissues were obtained following written informed consent. Preeclamptic pregnancies (term delivery $n=14$, pre-term delivery $n=8$ ) were diagnosed following American College of Obstetrics and Gynecology criteria. Control pregnancies (term delivery $n=20$, pre-term delivery $n=10$ ) were matched for gestational age, maternal age, maternal BMI, and baby weight. Pre-term delivery was defined as $<37$ completed weeks of gestation. Exclusion criteria for controls was hypertension (preexisting or onset during pregnancy) and/or proteinuria, as defined following American College of Obstetrics and Gynecology criteria. Participants' details are given in Table 1. Placentae were collected from a separate cohort of healthy term pregnancies $(n=11)$ for in vitro experiments. Tissues were collected as previously described ${ }^{27}$.

\section{Gene expression}

RNA was extracted using the RNeasy mini kit (Qiagen, Australia) and reverse transcribed using the iScript gDNA clear cDNA synthesis kit (Biorad, Australia), following manufactures' protocols. qPCR was performed using the Quanti-Nova SYBR Green PCR Kit (Qiagen) following manufacture's protocol. Expression of $S O D 1, S O D 2$, DMN1L, OPA1, BACE1, BID, PSENEN, CASP3, CASP8, $A D A M 10, X I A P$, and BCAP31 were analysed using Kiqstart SYBR Green primers (Sigma Life Science; primer pair one). Gene expression was normalised to the geometric mean of two loading controls (beta actin and beta-2 microglobulin; these genes were stable across all groups, within two standard deviations of the mean) and expression calculated using the $2^{-\Delta \Delta C t}$ method.

\section{Total protein extraction, mitochondrial fraction isolation, and protein estimation}

Approximately $30 \mathrm{mg}$ of tissue was homogenised in radioimmunoprecipitation buffer $(100 \mathrm{mM}$ Tris, $300 \mathrm{mM}$ $\mathrm{NaCl}, 10 \% \mathrm{NP}-40,10 \%$ sodium deoxycholate, $1 \%$ sodium dodecyl sulphate, protease inhibitor, $200 \mathrm{mM}$ phenylmethanesulfonyl fluoride) in a glass homogeniser then centrifuged at $13,200 \times g$ for 5 min and supernatant collected.

Crude mitochondrial enriched fractions were isolated based on published methodology ${ }^{28-30}$. Briefly, approximately $30 \mathrm{mg}$ of tissue was homogenised in isolation media ( $250 \mathrm{mM}$ sucrose, $0.5 \mathrm{mM} \mathrm{Na}_{2}$ EDTA, $10 \mathrm{mM}$ Tris, $\mathrm{pH}$ 7.4) then centrifuged at $1500 \times g$ for $10 \mathrm{~min}$. The 
Table 1 Patient parameters

\begin{tabular}{|c|c|c|c|c|c|c|}
\hline & \multicolumn{3}{|l|}{ Term } & \multicolumn{3}{|l|}{ Pre-term } \\
\hline & Control & Preeclamptic & & Control $^{a}$ & Preeclamptic & \\
\hline Gestational age (weeks) & $38.75 \pm 0.84$ & $37.88 \pm 2.10$ & NS & $29.29 \pm 3.83$ & $29.73 \pm 3.21$ & NS \\
\hline Maternal age (years) & $27.47 \pm 3.93$ & $29.70 \pm 7.73$ & NS & $28.23 \pm 6.29$ & $32.64 \pm 6.28$ & NS \\
\hline Maternal BMI $\left(\mathrm{kg} / \mathrm{m}^{2}\right)$ & $25.17 \pm 3.84$ & $27.39 \pm 5.83$ & NS & $28.88 \pm 7.72$ & $28.99 \pm 11.01$ & NS \\
\hline Baby weight (kg) & $3.26 \pm 0.36$ & $3.23 \pm 0.80$ & NS & $1.37 \pm 0.65$ & $1.25 \pm 0.63$ & NS \\
\hline $\mathrm{SBP}(\mathrm{mmHg})$ & $120.78 \pm 6.5$ & $149.30 \pm 7.5$ & $* * * *$ & $118.6 \pm 17.0$ & $167.36 \pm 17.6$ & $* * * *$ \\
\hline $\mathrm{DBP}(\mathrm{mmHg})$ & $74.44 \pm 6.2$ & $88.00 \pm 11.1$ & $* *$ & $71.5 \pm 14.7$ & $103.6 \pm 13.3$ & $* * * *$ \\
\hline IUGR (\%) & 0 & 9 & - & 50 & 38 & - \\
\hline Labour (\%) & $100 \%$ & $100 \%$ & - & $36 \%$ & $0 \%$ & - \\
\hline \multirow[t]{2}{*}{ Mode of delivery } & $100 \%$ VD & $38 \%$ VD & - & $36 \%$ VD & $0 \% \mathrm{VD}$ & - \\
\hline & $0 \%$ CS & $62 \% \mathrm{CS}^{\mathrm{b}}$ & - & $63 \%$ CS & $100 \%$ CS & - \\
\hline Sex of baby (male/female), $\%$ & $50 / 50$ & $44 / 55$ & - & $50 / 50$ & $63 / 37$ & - \\
\hline
\end{tabular}

Note: Data presented as mean \pm standard deviation

BM/body mass index, SBPsystolic blood pressure, DBPdiastolic blood pressure, VDvaginal delivery, CScaesarean section, NSnon-significant

${ }^{a}$ Reasons for pre-term delivery in the pre-term control group included pre-term premature rupture of the membranes, intrauterine growth restriction, hind water leak, hyperemesis, cholestasis, infection, and Factor V Leiden mutation

${ }^{\mathrm{b}}$ All term preeclamptic caesarean section deliveries were laboured

${ }^{* *} P<0.01, * * * * P<0.0001$

supernatant was centrifuged at $4000 \times g$ for $15 \mathrm{~min}$ to separate the mitochondrial pellet.

Protein content was determined using the Pierce BCA Protein Assay Kit (Thermo Fisher Scientific, Australia) following manufacture's protocol.

\section{Western blotting}

Protein was standardised to a concentration of $1 \mu \mathrm{g} / \mu \mathrm{L}$ in loading buffer $(62.5 \mathrm{mM}$ Tris $\mathrm{pH} 6.8,2 \%$ sodium dodecyl sulphate, $10 \%$ glycerol, $5 \% \beta$-mercaptoethanol, $0.001 \%$ bromophenol blue) and denatured by heating. $20 \mu \mathrm{g}$ of protein was loaded into $12 \%$ polyacrylamide gels for separation by electrophoresis, and proteins transferred onto polyvinylidene fluoride membranes (Merck, Australia). Membranes were blocked with Odyssey Blocking Buffer (Millennium Science, Australia) and incubated overnight with primary antibodies (Table 2). Membranes were incubated with secondary antibodies (1:10,000; IRDye 680 or IRDye $800 \mathrm{CW}$; LI-COR, Australia) for one hour and protein expression visualised using the Odyssey CLX (LI-COR). The level of the protein of interest was normalised to consistent protein loading control (beta actin, glyceraldehyde 3-phosphate dehydrogenase, or complex V).

\section{Hydrogen peroxide production}

Amplex Ultra Red (Thermo Fisher Scientific) was used to detect hydrogen peroxide $\left(\mathrm{H}_{2} \mathrm{O}_{2}\right)$ levels in protein lysates following the manufacture's protocol.
Table 2 Primary antibodies used in western blotting

\begin{tabular}{llll}
\hline $\begin{array}{l}\text { Protein } \\
\text { target }\end{array}$ & Manufacturer & $\begin{array}{l}\text { Catalogue } \\
\text { number }\end{array}$ & $\begin{array}{l}\text { Concentration } \\
\text { used }\end{array}$ \\
\hline DRP1 & Abcam & ab56788 & $1: 500-1: 1,000$ \\
FIS1 & GeneTex & GT4211 & $1: 1,000$ \\
OPA1 & Abcam & ab119685 & $1: 1,000$ \\
MFN1 & Abcam & ab57602 & $1: 1000$ \\
MFN2 & Abcam & ab56889 & $1: 200-1: 500$ \\
BAX & Abcam & ab32503 & $1: 500$ \\
BCL2 & Abcam & ab32124 & $1: 500$ \\
CASP9 & Bioss Antibodies & bs-0049R & $1: 1,000$ \\
Cleaved & Cell Signalling & Asp175 & $1: 1,000$ \\
CASP3 & & & \\
NFkB & Cell Signalling & D14E12 & $1: 1,000$ \\
ETS & Abcam & ab110411 & $1: 500-1: 1,000$ \\
complexes & & & $1: 1,000$ \\
$\beta$-actin & Abcam & ab8226 & $1: 2,500$ \\
GAPDH & Abcam & Ab9485 & \\
\hline
\end{tabular}

\section{Antioxidant activity}

Total antioxidant activity in protein lysates was measured following published methodology ${ }^{31,32}$. Briefly, protein lysates standardised to a concentration of $900 \mu \mathrm{g} / \mathrm{mL}$ 
or trolox standards were added to oxidised 2,2'-Azinodi-[3-ethylbenzthiazoline sulphonate] and reduction spectrophotometrically measured. Results are expressed relative to trolox activity ( $\mathrm{mmol} / \mathrm{L}$ trolox equivalent). Superoxide dismutase activity was determined in protein lysates using the Superoxide Dismutase Assay Kit (Cayman Chemical, Australia) following the manufacturer's protocol. Catalase activity was determined in protein lysates using the Catalase Activity Assay Kit (Abcam, Australia) following the manufacture's protocol. Glutathione peroxidase (GPx) activity was determined in protein lysates following published methodology ${ }^{33}$.

\section{Citrate synthase activity}

Citrate synthase activity was determined in protein lysates following published methodology ${ }^{34}$.

\section{MtDNA/nDNA ratio}

The mtDNA/nDNA ratio was measured as previously published $^{27}$. Briefly, qPCR was performed using mtDNA or nDNA primer sets (Table 3 ), and content calculated as the ratio of mtDNA to nDNA using the $2^{-\Delta \Delta C t}$ method.

\section{High-resolution respirometry}

Mitochondrial respiration was assessed as previously described $^{27}$. Briefly, the plasma membrane of tissue samples was permeabilized with $50 \mu \mathrm{g} / \mathrm{mL}$ saponin in $1 \mathrm{~mL}$ BIOPS solution (2.77 mM CaK ${ }_{2}$ EGTA, $7.23 \mathrm{mM}$ $\mathrm{K}_{2}$ EGTA, $5.77 \mathrm{mM} \mathrm{Na} \mathrm{NaTP}_{2}$, $6.56 \mathrm{mM} \mathrm{MgCl}_{2} \cdot 6 \mathrm{H}_{2} \mathrm{O}, 20$ $\mathrm{mM}$ taurine, $15 \mathrm{mM} \mathrm{Na}_{2}$ phosphocreatine, $20 \mathrm{mM}$ imidazole, $0.5 \mathrm{mM}$ dithiothreitol, $50 \mathrm{mM}$ MES, $\mathrm{pH}$ 7.1) for $30 \mathrm{~min}$ on ice, washed twice for $10 \mathrm{~min}$ on ice in MiR05 respiration medium $\left(0.5 \mathrm{mM}\right.$ EGTA, $3 \mathrm{mM} \mathrm{MgCl} 2 \cdot 6 \mathrm{H}_{2} \mathrm{O}$, $60 \mathrm{mM}$ K-lactobionate, $20 \mathrm{mM}$ taurine, $10 \mathrm{mM} \mathrm{KH}{ }_{2} \mathrm{PO}_{4}$, $20 \mathrm{mM}$ HEPES, $110 \mathrm{mM}$ sucrose, $1 \mathrm{~g} / \mathrm{L}$ BSA essentially fatty acid free, $\mathrm{pH}$ 7.1) and blot-dried before measuring wet weight. To measure mitochondrial respiration, $10-20 \mathrm{mg}$ wet weight of tissue was added to an

Table 3 Primer sequences for mitochondrial content determination

\begin{tabular}{llll}
\hline Target & Name & Primer sequence & Reference \\
\hline nDNA & MTAIB_f & GAG TाT CCT GGA CAA ATG AG & 69 \\
& MTAIB_r & CAT TGT TTC ATA TCT CTG GCG & \\
nDNA & MTBA_f & AGC GGG AAA TCG TGC GTG AC & 69 \\
& MTBA_r & AGG CAG CTC GTA GCT CTT CTC & \\
mtDNA & MTRT4_f & ATG GCC CAC CAT AAT TAC CC & 69 \\
& MTRT4_r & CAT TाT GGT TCT CAG GGT TTG & \\
mtDNA & MTRT5_f & GCC TTC CCC CGT AAA TGA TA & 70 \\
& MTRT5_r & TTA TGC GAT TAC CGG GCT CT & \\
\hline
\end{tabular}

Oxygraph-2k respirometer (Oroboros Instruments, Austria) chamber containing MiR05 at $37^{\circ} \mathrm{C}$. The activity of specific respiratory states was determined by the addition of ETS substrates and inhibitors; pyruvate $(5 \mathrm{mM})$, glutamate $(10 \mathrm{mM})$, and malate $(2 \mathrm{mM})$ were added to determine complex I (CI) mediated LEAK respiration, then oxidative phosphorylation (OXPHOS) through CI was stimulated by the addition of ADP (1-5 mM). Cytochrome $\mathrm{c}(10 \mu \mathrm{M})$ was then added to test the integrity of the outer mitochondrial membrane. Succinate $(10 \mathrm{mM})$ addition then stimulated OXPHOS through complex II (CII), and this was followed by titration of the uncoupler carbonyl cyanide m-chloro phenyl hydrazone (CCCP; 1 $\mathrm{mM}$ ) to investigate ETS capacity with CII mediated flux alone. Rotenone $(1 \mu \mathrm{M})$ was added to inhibit CI. The addition of antimycin A inhibited complex III (CIII), and residual oxygen consumption was measured (indicating non-ETS respiration). $\mathrm{N}, \mathrm{N}, \mathrm{N}^{\prime}, \mathrm{N}^{\prime}$-tetramethyl-p-phenylenediamine dihydrochloride (TMPD; $0.5 \mathrm{mM}$ ) and ascorbate $(2 \mathrm{mM})$ were added to determine OXPHOS through complex IV.

\section{In vitro hypoxia/reoxygenation with measurement of mitochondrial respiration and hydrogen peroxide production}

Samples from the same placenta were assessed simultaneously, with one sample maintained at atmospheric oxygen while the other was allowed to respire in anoxia. Anoxia was maintained for 20 min before reintroduction of oxygen. Amplex Ultra Red (Thermo Fisher Scientific) was used to detect $\mathrm{H}_{2} \mathrm{O}_{2}$ levels in placental tissue during respiration following published methodology ${ }^{35}$.

\section{Statistical analysis}

All values are mean \pm SD. PCR and western blot data is presented normalised to healthy term controls. For activity assays, PCRs and western blots, effect of preeclampsia, gestational age and the interaction of these factors was determined with two-way ANOVA with Tukey's multiple comparisons test. For western blots, representative samples from term and pre-term groups were included in each experiment within gestational groups, but not between gestational groups. For respirometry, normality was met and the effect of preeclampsia was determined with $t$-test. Effect of hypoxia/reperfusion was determined with paired $t$-test, or Wilcoxon test if normality was not met. Significance level was set at 0.05 . Analyses were performed using GraphPad PRISM 7.02 (GraphPad, USA).

\section{Results \\ Hydrogen peroxide production and signalling response \\ To investigate ROS, antioxidants, and signalling responses in placentae from preeclamptic pregnancies}


that reached term versus those that delivered pre-term, $\mathrm{H}_{2} \mathrm{O}_{2}$ production, antioxidant function, and ROS-related signalling were assessed in matched placentae from control and preeclamptic pregnancies (Table 1; Fig. 1). Preeclampsia and gestational age affected both $\mathrm{H}_{2} \mathrm{O}_{2}$ production and total antioxidant activity. In placentae from term preeclamptic pregnancies, there was an increase in total antioxidant activity compared to controls, whereas a decrease in $\mathrm{H}_{2} \mathrm{O}_{2}$ production and no change in total antioxidant activity were observed in pre-term preeclamptic placentae compared to controls (Fig. 1a, b).

Preeclampsia and gestational age affected markers of superoxide dismutase (SOD) function (Fig. 1c-f). There were no changes in markers of SOD function between healthy term and preeclamptic term placentae. In preterm preeclamptic placentae, the expression of the mitochondrial-targeted SOD2 was not changed; however, SOD activity in enriched mitochondrial fractions was reduced in pre-term preeclamptic placentae compared to controls (Fig. 1e, f). Catalase activity (fold change 0.467; Fig. 1g) and glutathione peroxidase (GPx; fold change 0.422 ; Fig. 1h) activity were decreased in pre-term compared to term placentae. GPx activity was increased in placentae from term preeclamptic pregnancies compared to controls.

Levels of the immune and apoptotic regulator nuclear factor kappa-light-chain-enhancer of activated B cells (NF-KB) were unchanged in term preeclamptic placentae and increased in pre-term preeclamptic placentae compared to controls (Fig. 1i). The $\alpha$-secretase ADAM metallopeptidase domain 10 (ADAM10; Fig. 1j), $\beta$-secretase 1 (BACE1) and presenilin enhancer, gammasecretase subunit (PSENEN) were not different between groups (Supplementary Fig. 1).

\section{Mitochondrial fission/fusion}

To investigate if the response of placental mitochondria to preeclampsia was different between term and pre-term pregnancies, modulators of mitochondrial fission/fusion processes were investigated (Fig. 2a). There were no preeclampsia-associated changes in pro-fission dynamin1-like protein $(D M N 1 L / D R P 1)$ at the gene (Fig. 2b) or protein (Fig. 2c and d) level in any groups. In term preeclamptic placentae, there was a decrease in fission 1 protein (FIS1) compared to controls (Fig. 2e). Pro-fusion mitochondrial dynamin like GTPase (OPA1) expression was affected by gestational age and interaction of preeclampsia/gestational age (Fig. 2f). There was no change in OPA1 total protein levels (Supplementary Fig. 2), but a relative increase in OPA1 long isoform (L-OPA1) compared to the short isoform (S-OPA1; Fig. 2g), and profusion mitofusin 1 (MFN1) levels were increased (Fig. 2h), with no change in mitofusin 2 (MFN2), in term preeclamptic placentae compared to controls (Fig. 2i).

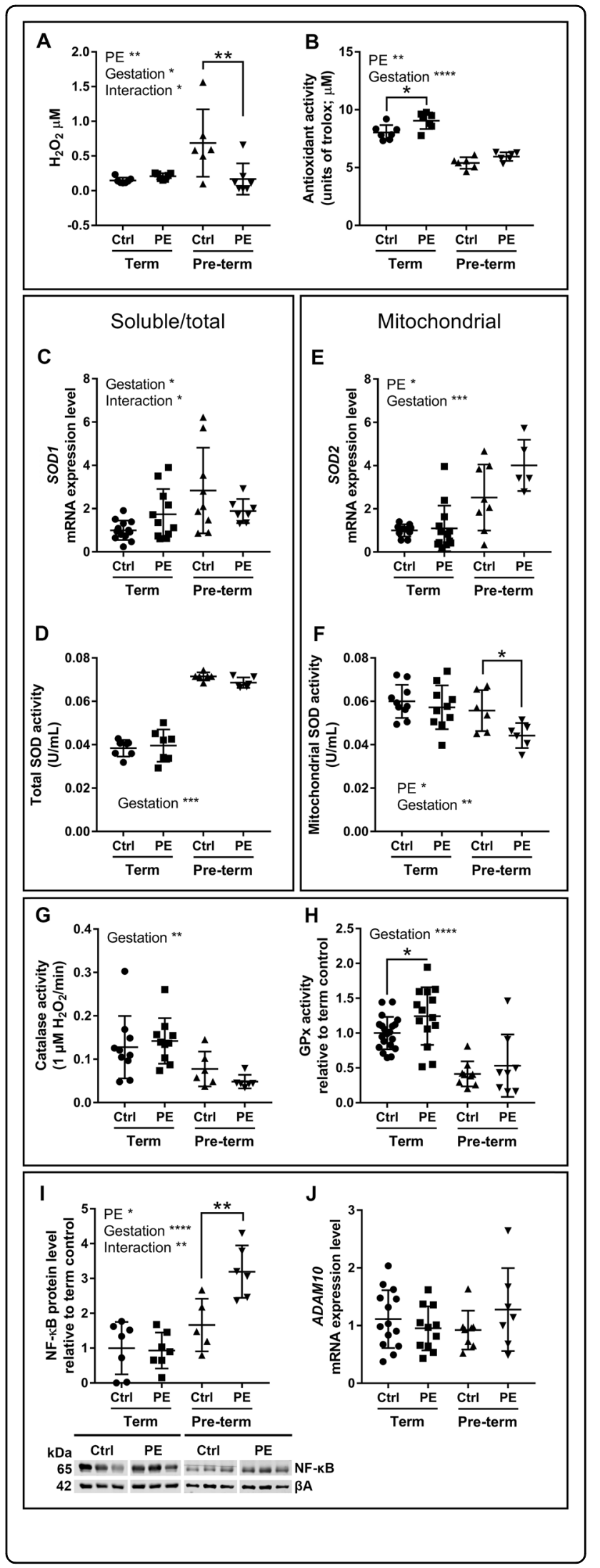


Fig. 1 Total antioxidant activity increases in term preeclamptic placentae and superoxide dismutase function is reduced in preterm preeclamptic placentae. a Hydrogen peroxide $\left(\mathrm{H}_{2} \mathrm{O}_{2}\right)$ level was affected by preeclampsia $(p=0.0295)$, gestation $(p=0.0186)$ and interaction factor $(p=0.0074)$, with levels decreased in pre-term preeclamptic placentae compared to pre-term controls ( $p=0.0014$, fold change 0.244$)$. $\mathbf{b}$ Total antioxidant activity was affected by preeclampsia $(p=0.0024)$ and gestation $(p<0.0001)$, with activity increased in term preeclamptic placentae compared to term controls ( $p=0.0171$, fold change 1.130). c Soluble superoxide dismutase 1 (SOD1) mRNA expression was affected by gestation $(p=0.0130)$, and the interaction of preeclampsia/gestation $(p=0.0329)$. $\mathbf{d}$ Total superoxide dismutase (SOD) activity over all cellular compartments was affected by gestation $(p<0.0001)$. e Mitochondrial superoxide dismutase 2 (SOD2) mRNA expression was affected by preeclampsia $(p=0.0310)$ and gestation $(p=0.0001)$. $\mathbf{f}$ SOD activity in enriched mitochondrial fractions was affected by preeclampsia $(p=0.0305)$ and gestation $(p=0.0100)$, and was reduced in pre-term preeclamptic placentae compared to pre-term controls ( $p=0.0277$, fold change 0.794). $\mathbf{g}$ Catalase activity was affected by gestation ( $p=0.0010)$. h Glutathione peroxidase (GPx) activity was affected by gestation $(p<0.0001)$ and increased in term preeclamptic placentae compared to term controls ( $p=0.0357$, fold change 1.244). i Nuclear factor kappa-light-chain-enhancer of activated B cells (NF-kB) was affected by preeclampsia $(p=0.0164)$, gestation $(p<0.0001)$, and interaction factor $(p=0.0095)$, and was increased in pre-term preeclamptic placentae ( $p=0.0077$, fold change 1.599). $\mathbf{j}$ mRNA expression of ADAM metallopeptidase domain 10 (ADAM10) was not different between groups. $\beta$ Abeta actin. Representative western blot images. $N=5-20 ;{ }^{*} P<0.05 ;{ }^{*} P<0.01 ;{ }^{* *} P<0.001$

\section{Mitochondrial-linked apoptotic signalling}

As mitochondrial processes are closely linked to the coordination of apoptosis (Fig. 3a), apoptotic related signalling molecules were investigated (Fig. 3). There were no changes in levels of the execution-phase apoptotic protein cleaved caspase 3 (CASP3; Fig. 3b), and also no changes in CASP3 mRNA expression (Fig. 3h) between preeclamptic placentae and controls. CASP3 was affected by gestational age, and the interaction of preeclampsia/gestational age (Fig. 3b). Proapoptotic Bcl-2-associated X protein (BAX; Fig. 3c) was reduced and anti-apoptotic B-cell lymphoma 2 (BCL2; Fig. 3d) was increased in term preeclamptic placentae compared to term controls. The BAX/BCL2 ratio was reduced in in term preeclamptic placentae compared to term controls, and increased in pre-term preeclamptic placentae compared to pre-term controls (Fig. 3e). There were no changes in the mitochondrial-linked apoptotic protein cleaved caspase 9 (CASP9; Fig. 3f) or expression of the endoplasmic reticulum (ER) protein B-cell-receptor-associated protein 31 (BAP31; Supplementary Fig. 3). Expression of the anti-apoptotic $\mathrm{X}$-linked inhibitor of apoptosis protein $(X I A P)$, and pro-apoptotic caspase 8 (CASP8) and $\mathrm{BH} 3$ interactingdomain death agonist $(B I D)$ were affected by gestation (Fig. 3i, j, k, respectively).

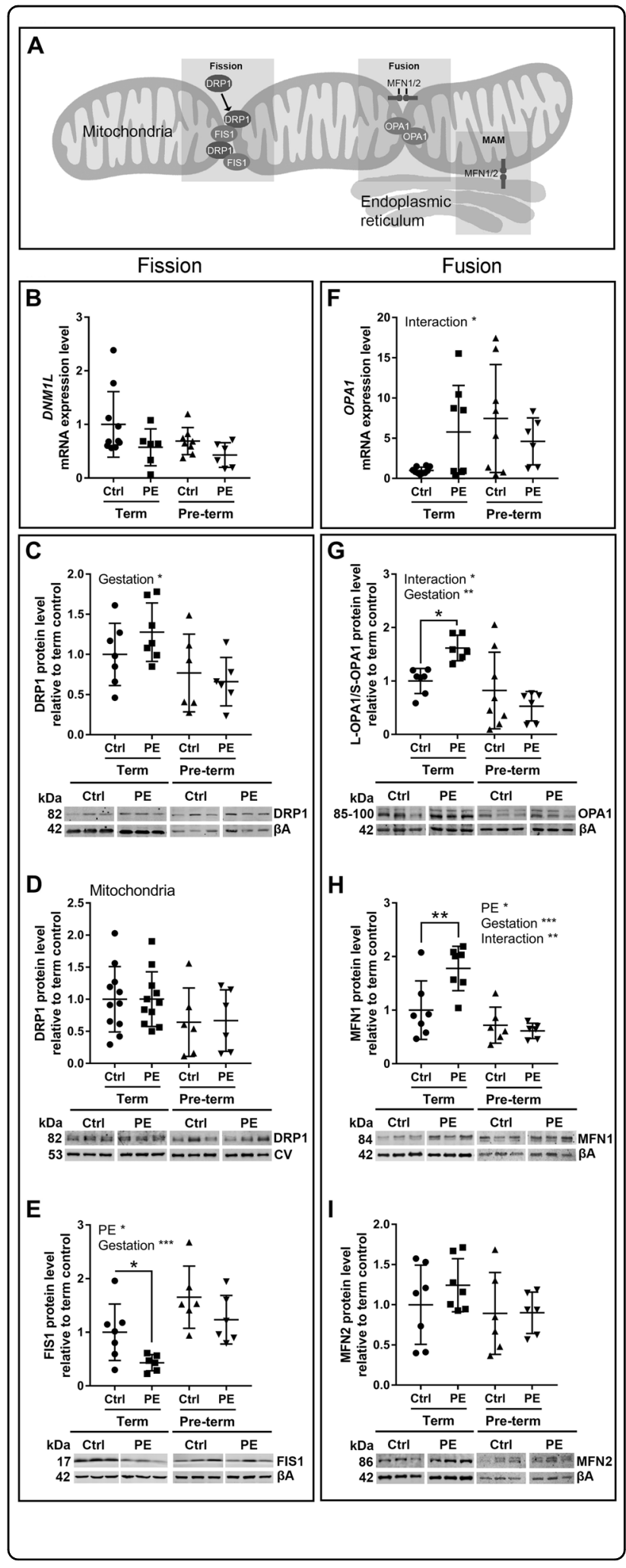

Mitochondrial complexes, respiration, and content

The relative proportions of mitochondrial complexes (CI-CV) can influence ETS efficacy. Complexes II 
Fig. 2 Mitochondrial fission/fusion signalling is disrupted differentially in term and pre-term preeclamptic placenta. a Diagram of proteins involved in mitochondrial fusion/fission processes. b mRNA expression, (c) total protein and (d) mitochondrial protein fractions of pro-fission dynamin-1-like protein (mRNA = $D N M 1 L$; protein $=$ DRP1) were unchanged between control and preeclamptic placentae, with DRP1 affected by gestation ( $p=0.0122)$. e Mitochondrial fission 1 protein (FIS1) was affected by preeclampsia $(p=0.0145)$ and gestation $(p=0.0008)$, and was decreased in term preeclamptic placentae ( $p=0.0384$, fold change 0.406$)$. f mRNA expression of pro-fusion mitochondrial dynamin like GTPase (OPA1 /OPA1) was affected by the interaction of preeclampsia and gestation $(p=0.0288)$. $\mathbf{g}$ The ratio of the OPA 1 long isoform to short isoform ( $L-$ OPA1/S-OPA1) was affected by gestation $(p=0.0013)$ and interaction factor $(p=0.0147)$, and was increased in term preeclamptic placentae ( $p=0.0207$, fold change 1.617) and unchanged in pre-term preeclamptic placentae. $\mathbf{h}$ Mitofusin 1 (MFN1) was affected by preeclampsia $(p=0.0436)$ and gestation $(p=0.0001)$ and interaction factor $(p=0.0099)$, and was increased in term preeclamptic placentae ( $p=0.007$, fold change 1.778), (i) with no difference in mitofusin 2 (MFN2). MAMmitochondria associated membrane; $\beta$ Abeta actin; $C V$ mitochondrial complex $\mathrm{V}$. Representative western blot images. $N=6-7 ;{ }^{*} P<0.05 ;{ }^{*} P<0.01 ;{ }^{* *} P<0.001$

and III were increased in term preeclamptic placentae, but unchanged in pre-term preeclamptic placentae, compared to controls (Fig. 4a and b). Citrate synthase activity relates to mitochondrial function/content, and was not different between preeclamptic placentae and controls, but affected by gestational age (Fig. 4c). The mtDNA/nDNA ratio estimates mitochondrial content, and was increased in term preeclamptic placentae compared to controls $(p=0.0093$, fold change 1.458). There was no difference in total placental weight in term preeclamptic placentae compared to controls (control $0.62 \pm 0.11 \mathrm{~kg}$, preeclamptic $0.68 \pm 0.11 \mathrm{~kg}$ ).

The production of ATP is achieved through oxidative phosphorylation (OXPHOS) via ETS complexes. To better characterise the effect of changes in relative proportions of mitochondrial complexes in term preeclamptic placentae, mitochondrial respiration was examined in these tissues. There was an overall increase in mitochondrial respiration in term preeclamptic placentae compared to controls (Fig. 4d). Oxidative phosphorylation through mitochondrial complexes I (OXPHOS I) and I + II, and non-phosphorylating LEAK respiration, were increased in term preeclamptic placentae compared to controls. OXPHOS I as a proportion of maximal respiratory capacity was increased in term preeclamptic placentae, and reserve capacity (measure of mitochondrial ability to respond to increased energy demand) was reduced in term preeclamptic placentae compared to controls (Fig. 4d).

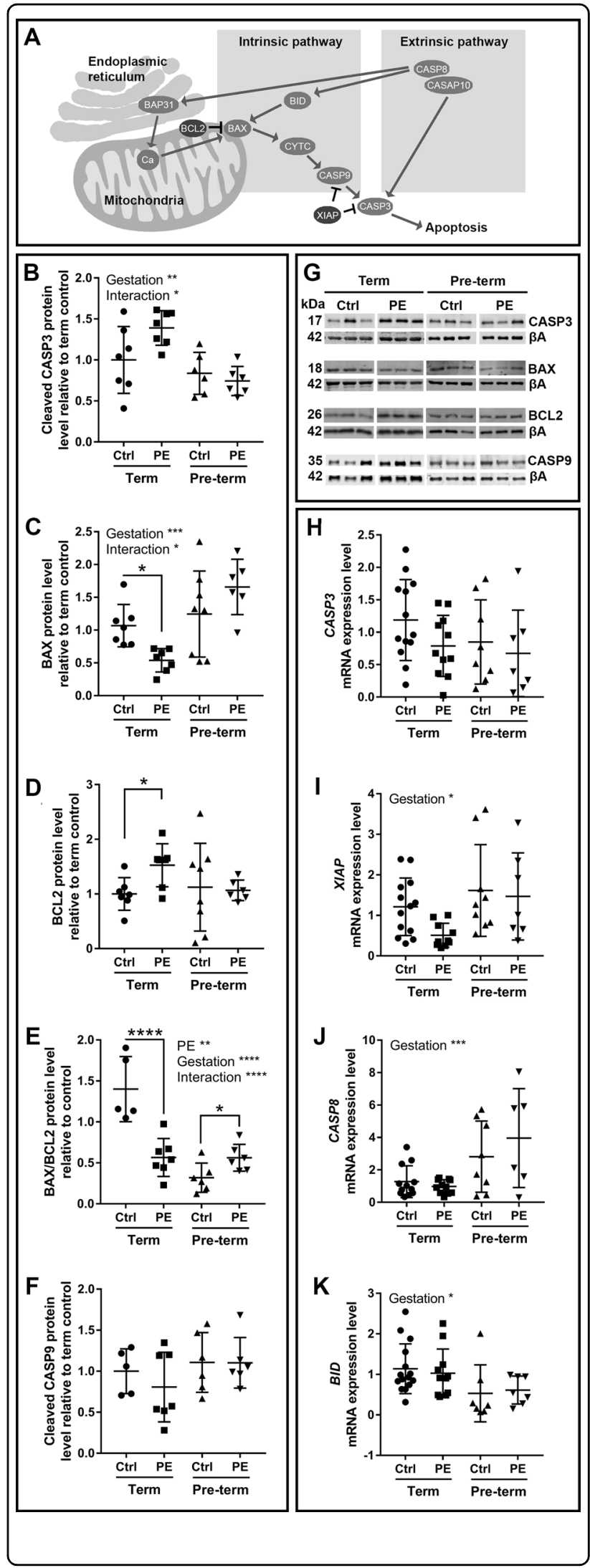


Fig. 3 Mitochondrial apoptotic signalling is disrupted differentially in term and pre-term preeclamptic placenta. a Diagram of proteins involved in mitochondrial apoptotic signalling. b Pro-apoptotic cleaved caspase 3 (CASP3) was affected by gestation $(p=0.0014)$ and interaction factor $(p=0.0403)$. c Pro-apoptotic Bcl-2associated $X$ protein (BAX) was affected gestation $(p=0.0008)$ and interaction factor $(p=0.0101)$, and was reduced in term preeclamptic placentae ( $p=0.0356$, fold change 0.5394$)$. $\mathbf{d}$ Anti-apoptotic B-cell lymphoma 2 (BCL2) was increased in term preeclamptic placentae ( $p=0.0156$, fold change 1.526). e The BAX/BCL2 ratio was affected by preeclampsia $(p=0.0093)$, gestation $(p<0.0001)$ and interaction factor $(p<0.0001)$, and was decreased in term preeclamptic placentae $(p<0.0001$, fold change 0.4044$)$ and increased in pre-term preeclamptic placentae ( $p=0.0334$, fold change 1.763). f Proapoptotic cleaved caspase 9 (CASP9) did not change. $\mathbf{g}$ Representative western blot images. $\mathbf{h}$ mRNA expression of CASP3 was not different between groups. mRNA expression of (i) X-linked inhibitor of apoptosis protein $(X I A P)$, (j) caspase 8 (CASP8), and (k) BH3 interactingdomain death agonist $(B I D)$ were affected by gestation $(p=0.0172$, $0.0004,0.0136$, respectively). BAP31B-cell-receptor-associated protein 31; Cacalcium; CASP10caspase 10; CYTCcytochrome c; $\beta$ Abeta actin. $N=6-8 ;{ }^{*} P<0.05 ;{ }^{*} P<0.01 ;{ }^{*} P<0.001$

Increased mitochondrial respiration and production of hydrogen peroxide post in vitro hypoxia/reoxygenation

To investigate the direct effect of hypoxia/reoxygenation on placental tissue, healthy term placentae were subjected to hypoxia followed by reoxygenation in vitro while measuring mitochondrial respiration and $\mathrm{H}_{2} \mathrm{O}_{2}$ production. Similar to that observed in term preeclamptic placentae (Figs. 1a and $4 \mathrm{c}$ ), there was an increase in $\mathrm{H}_{2} \mathrm{O}_{2}$ production and mitochondrial respiration post hypoxia/reoxygenation in healthy placentae compared to matched tissue that did not undergo hypoxia/reoxygenation (Fig. 5). OXPHOS I + II, LEAK respiration, and maximum capacity of the respiratory system, were increased in healthy placental tissue after hypoxia/reoxygenation, reserve capacity was not changed (Fig. 5a). Hypoxia/reoxygenation of healthy placental tissue did not change $\mathrm{H}_{2} \mathrm{O}_{2}$ production during OXPHOS I + II, but $\mathrm{H}_{2} \mathrm{O}_{2}$ production was increased during LEAK respiration (Fig. 5b).

\section{Discussion}

Preeclampsia is a dangerous pregnancy disorder with symptoms that range in severity ${ }^{5}$. The broad symptomatic range may represent different aetiologies/progression paths leading to different outcomes ${ }^{4}$. This study aimed to characterise key pathways that may contribute to disease severity by investigating placental adaptations in preeclamptic pregnancies that successfully reached term delivery compared to those that did not. Placentae from term preeclamptic pregnancies displayed multiple adaptations in mitochondrial function and related processes that were only minimally observed in preeclamptic pregnancies that delivered pre-term. In contrast, pre-term preeclamptic placentae showed reduced antioxidant function, and differences in immune and apoptotic signalling. Further, we show that hypoxia/reoxygenation of healthy tissue leads to direct and rapid changes in placental mitochondrial function that are similar to those observed in placentae from term preeclamptic pregnancies. Our findings suggest that the placental adaptations that determine disease severity and allow pregnancies to progress to term involve antioxidant responses and dynamic mitochondrial-related processes.

\section{An increased antioxidant response is associated with preeclamptic placentae that reach term}

Oxidative stress is a feature of disorders related to ischaemia/reperfusion injury ${ }^{36}$. Preeclampsia is thought to be associated with placental ischaemia/reperfusion, and increased markers of oxidative stress have been measured in preeclampsia ${ }^{6,7,19,37,38}$. In the current work, we have found that placentae from term preeclamptic pregnancies had increased levels of the $\operatorname{ROS} \mathrm{H}_{2} \mathrm{O}_{2}$, but they also appear to have a compensatory antioxidant response, with increased total antioxidant activity that may be partly driven through increased GPx function. In contrast, placentae from pre-term preeclampsia did not exhibit this antioxidant response, with reduced SOD function, as well as evidence of a greater immune response, with increased NF- $\kappa \mathrm{B}$. This decreased antioxidant function and increased immune response in more severe preeclampsia is in agreement with previously published data ${ }^{19,39-43}$. The decreased mitochondrial SOD activity in pre-term preeclamptic placentae may have led to the lower levels of $\mathrm{H}_{2} \mathrm{O}_{2}$ in these tissues compared to pre-term controls, as SOD converts superoxide to $\mathrm{H}_{2} \mathrm{O}_{2}$. However, a portion of the control pre-term pregnancies experienced labour (compared to none of the pre-term preeclamptic pregnancies; Table 1), and the relative increase in $\mathrm{H}_{2} \mathrm{O}_{2}$ may also be related to this factor. Further, it is possible that the differences in antioxidant function over gestation (decreased total SOD activity, and increased catalase, GPx and total antioxidant activity at term) are part a normal progression to delivery, or are due to the complications of the pre-term pregnancies. Additionally, tissues used in this investigation came from an overweight population (Table 1). Although body mass index was not different between the control and preeclamptic populations in this study, high adiposity is a known risk factor for preeclampsia, and the outcomes observed here may be different in a lean population. In term preeclampsia there was also no change in the ROS-responsive secretase $A D A M 10^{44}$. $A D A M 10$ has previously been observed to increase in pre-term preeclampsia, and through its action in protein secretion may contribute to increased placental shedding of soluble fms-like tyrosine kinase 1 (sFlt1); 


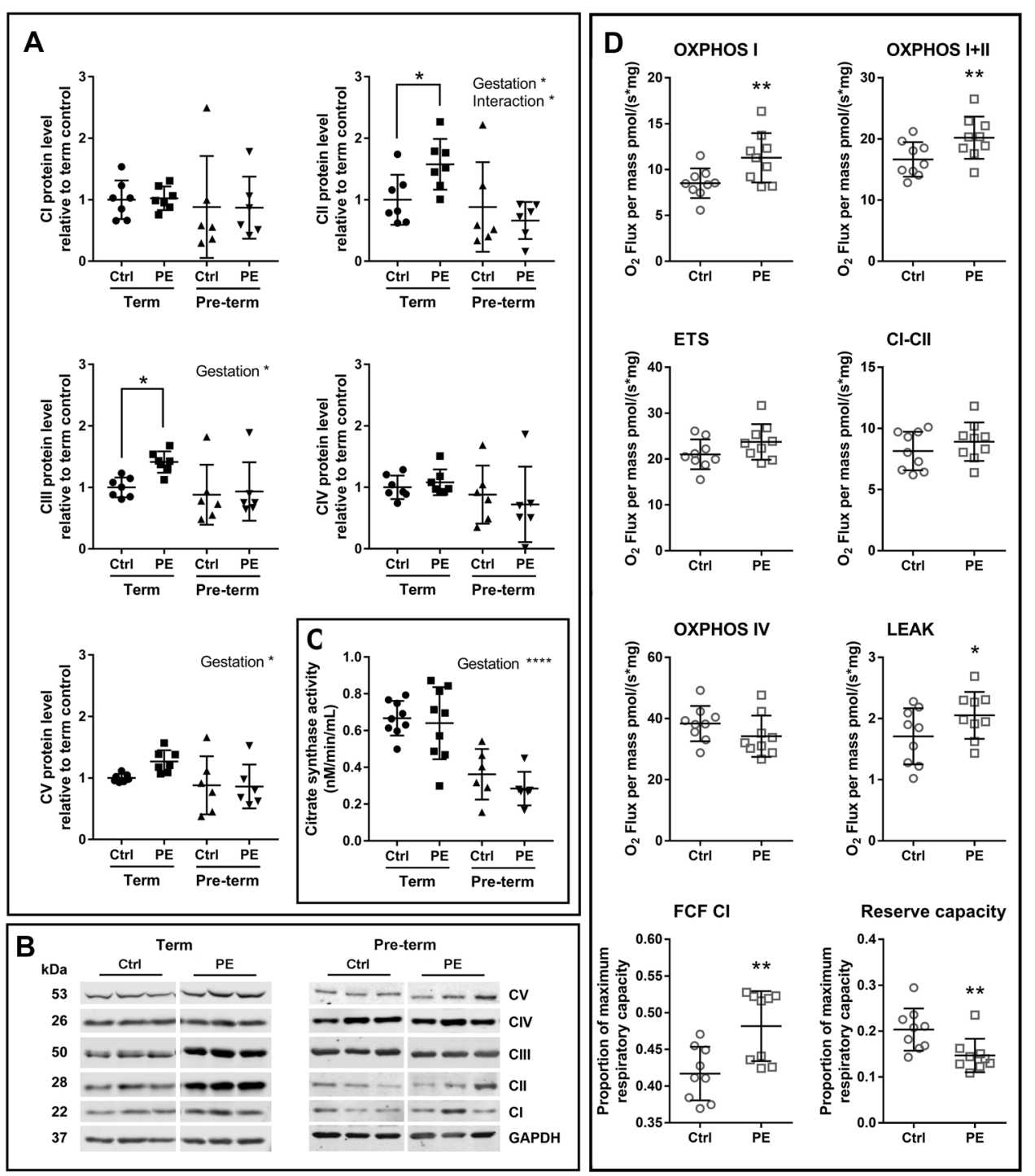

Fig. 4 Mitochondrial complex levels and respiration are dysregulated in preeclamptic placentae. a Protein levels of mitochondrial complexes II and III were increased in preeclamptic placentae from term pregnancies compared to control placentae from term pregnancies $(p=0.0360,0.0369$, fold change 1.576, 1.413, respectively). Complex II was affected by gestation $(p=0.0125)$ and interaction factor $(p=0.0478)$, complex III was affected by gestation $(p=0.0401)$, and complex $V$ (ATP synthase) was affected by gestation $(p=0.0370)$. There was no change in the protein level of complexes I, IV and V in placentae from term pregnancies, or all complexes in placentae from pre-term pregnancies. b Representative western blot images of mitochondrial complexes. c Citrate synthase activity was affected by gestation $(p<0.0001$, fold change 0.495 pre-term compared to term pregnancies). d Oxidative phosphorylation through mitochondrial complexes I (OXPHOS I) and I + II, and non-phosphorylating LEAK respiration, were increased in term preeclamptic placentae $(p=0.0084, p=0.0199, p=0.0310$, fold change $1.188,1.232,1.150$, respectively). Maximum capacity of the respiratory system (ETS), oxygen consumption through complex II (CI-CII) and OXPHOS IV were not different. OXPHOS I as a proportion of maximal respiratory capacity (FCF CI) was increased in term preeclamptic placentae ( $p=0.0026$, fold change 1.126). Reserve capacity was reduced in term preeclamptic placenta $(p=0.0042$, fold change 0.745$)$. GAPDH $=$ Glyceraldehyde 3-phosphate dehydrogenase. $N=6-10$; ${ }^{*} P<0.05$; ${ }^{* *} P<0.01$, ***P $<0.001$

a factor thought to play an important role in preeclampsia pathogenies ${ }^{45-48}$. The lack of change found in ADAM10 in the current investigation suggests that term preeclamptic pregnancies may not exhibit the same level of protein misfolding observed in pre-term tissue ${ }^{45}$. The ability to counter ROS may therefore represent a successful adaptation of placentae from the pregnancies with preeclampsia that reach term, allowing the placenta to continue to function under adverse conditions. Indeed, in term pregnancies complicated by maternal asthma, placental enzymatic antioxidant capacity is increased ${ }^{49}$, suggesting a compensatory response to oxidative stress occurred in placentae from these otherwise healthy pregnancies. 


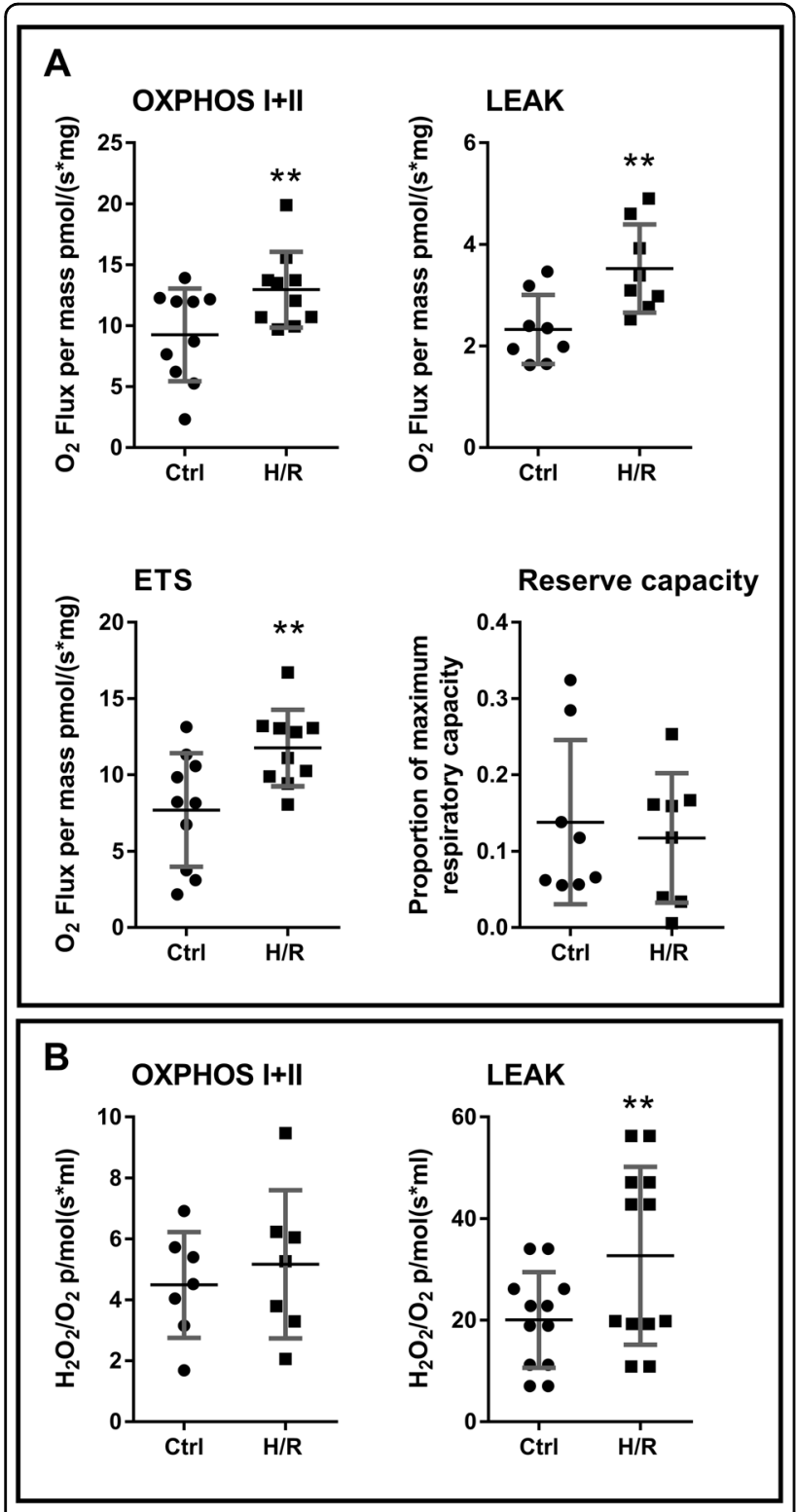

Fig. 5 In vitro hypoxia/reoxygenation of placental tissue leads to increased mitochondrial respiration and increased production of hydrogen peroxide during LEAK state respiration. a Oxidative phosphorylation through mitochondrial complexes I + II (OXPHOS I + II), non-phosphorylating LEAK respiration, and maximum capacity of the respiratory system (ETS), were increased in healthy placental tissue after hypoxia/reoxygenation ( $p=0.0065, p=0.0091, p=0.0060$, fold change $1.401,1.516,1.527$, respectively). Reserve capacity was not changed. $\mathbf{b}$ Hydrogen peroxide production was not changed during OXPHOS I+ II, and was increased in LEAK respiration in healthy placental tissue after hypoxia/reoxygenation $(p=0.0020$, fold change 1.945). $N=11 ;{ }^{* *} P<0.01$

Mitochondrial-related adaptations: fission and fusion

ROS signalling and mitochondrial function may be important in the response of preeclamptic placentae that reach term. Mitochondria undergo constant morphological change through cycles of fission and fusion, which are part of the mitochondrial response to environmental conditions, including stress ${ }^{9,50,51}$. These adaptive processes likely contribute to whether a tissue responds to minimise damage, or progresses to a more pathological state. The mitochondrial fission/fusion process was differentially disrupted in term and pre-term preeclamptic placentae, indicating that distinct cellular damage/repair pathways are activated in the different forms of the disorder. A pro-fusion environment was present in term preeclamptic pregnancies, with decreased pro-fission FIS1 and increased pro-fusion L-OPA1 and MFN1, whereas no changes in fission/fusion regulator proteins were observed in pre-term preeclamptic pregnancies. This contrasts other studies, which have reported increases in the pro-fission regulator OPA1 and decreases in the pro-fusion regulator DRP1 in early onset preeclampsia $^{52,53}$. However, other fission/fusion regulators including mitochondrial fission factor ${ }^{53}$, MFN1 and $M F N 2^{52}$, were not found to be changed in preeclamptic placentae in these studies, suggesting that the regulation of mitochondrial fission/fusion in the placenta is complex. Fission allows sequestering of damaged mitochondria for disposal by autophagy, whereas fusion can allow recovery of damage by fusing lower functioning mitochondria to better functioning parts of the reticular network ${ }^{9}$. Therefore, mitochondrial fusion may also have contributed to the ongoing successful function of the placenta in preeclamptic pregnancies that reached term.

\section{Mitochondrial-related adaptations: apoptosis}

Preeclampsia has been associated with increased markers of apoptosis in trophoblasts/the placenta ${ }^{41,54-57}$, and we also observed an increase in the pro-apoptotic BAX/ BCL2 ration in pre-term preeclamptic placentae. In placenta from term preeclampsia, we found a decrease in pro-apoptotic BAX and an increase in anti-apoptotic BCL2, as well as no change in CASP9, demonstrating active mitochondrial suppression of apoptotic signalling and suggesting that mitochondria promote cell survival in these placentae.

Although we observed evidence of mitochondrial apoptotic signalling suppression, other mechanisms may signal apoptosis. Mitochondrial/ER crosstalk is important in stress mediated apoptosis ${ }^{58}$. We found no preeclampsia-related changes in BAP31, CASP8 or BID, which can signal apoptosis through the extrinsic pathway and via the ER. Apoptotic signalling in preeclamptic placentae may be regulated by ER associations ${ }^{59}$, but determining the level of ER involvement in preeclampsia requires additional investigation. Further, the outer layer of the human placenta is a multinuclear syncytium (the syncytiotrophoblast), in which the apoptotic cascade may have specific functions in the progression of cell fate ${ }^{60}$. 
Mitochondrial-related adaptations: respiration and content

Given the evidence of mitochondrial salvage in placentae from term preeclampsia (discussed above), we investigated if these mitochondria functioned normally. The organisation of ETS complexes determines mitochondrial energy efficiency ${ }^{61,62}$. We observed changes in the relative levels of ETS complexes in term preeclamptic placentae, suggesting that there may be effects on mitochondrial efficiency. There was no change in citrate synthase activity between control and preeclamptic placentae, suggesting overall mitochondrial tissue function was not altered in preeclamptic placentae. Of note, citrate synthase activity was reduced in pre-term relative to term tissue, suggesting that placental mitochondrial function changes over this gestational period. Indeed, we have previously shown that mitochondrial respiration changes over early gestation ${ }^{27}$. However, all pre-term tissue is necessarily from complicated pregnancies, and pathology in the pre-term control group may impact on mitochondrial related functions. Future work will be required to investigate if there are changes in mitochondrial respiration and other functions in pre-term tissues. Term preeclamptic tissues had higher mitochondrial respiratory levels; however, there was a decrease in the reserve capacity, indicating mitochondria are routinely functioning closer to their maximum capacity. Changes in ETS complex levels could disrupt the formation of mitochondrial supercomplexes and result in increased production of $\operatorname{ROS}^{63}$. Therefore, increased respiration through a dysfunctional ETS may have led to the increased levels of $\mathrm{H}_{2} \mathrm{O}_{2}$ observed in preeclamptic tissue. Mitochondrial respiration can be increased in skeletal muscle in response to exercise as an adaptation to greater energy demands ${ }^{64}$, and the increase in respiration observed in preeclamptic placentae in the current study may represent a mechanism by which the tissue is able to maintain overall function with suboptimal mitochondrial function.

\section{Mitochondrial respiration and reactive oxygen species production increase after hypoxia/reoxygenation}

The pathophysiology of preeclampsia is thought to be associated with aberrant maternal blood flow to the placenta $^{6,7}$. Disrupted oxygen levels have been proposed to lead to placental dysfunction ${ }^{6}$, but may also be a trigger for the adaptations observed in the current study. In vitro exposure of healthy placental tissue to hypoxia and reoxygenation leads to a rapid increase in respiration, demonstrating that placental mitochondria are capable of responding to variations in oxygen supply even over short time periods. Indeed, we have previously shown that labour is associated with increases in placental mitochondrial respiration ${ }^{27}$. Others have observed increased markers of oxidative stress in laboured tissue ${ }^{65-68}$, suggesting a direct link between placental mitochondrial respiration and oxidative damage. In the current investigation, we also found an increase in $\mathrm{H}_{2} \mathrm{O}_{2}$ production with hypoxia/reoxygenation, demonstrating that hypoxia/ reoxygenation can be a direct source of ROS, which may be both damaging or act in signalling.

\section{Conclusions}

We show multiple mitochondrial-related adaptions in placentae from preeclamptic pregnancies that reached term, which are not found in preeclamptic pregnancies that delivered pre-term (Fig. 6). ROS signalling in term preeclamptic placentae may be at a level to trigger compensatory antioxidant and mitochondrial responses, allowing tissue level maintenance of function when there is organelle level dysfunction. In other tissues, mitochondrial ROS signalling is known to lead to a range of physiological responses (including increased antioxidant

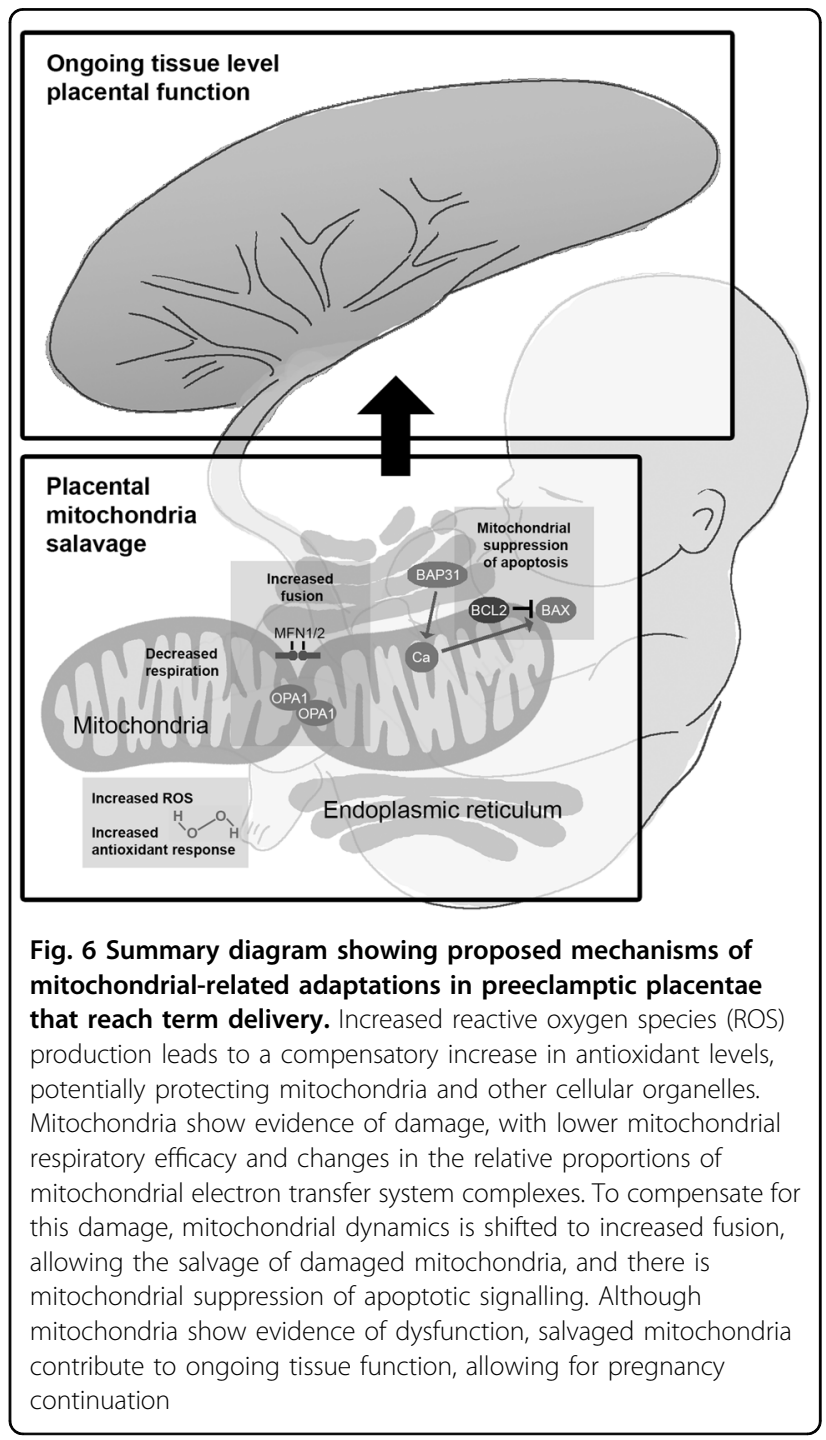


defences, activation of potassium channels, activation of uncoupling proteins, and expression of pro-survival genes) that can precondition improved cell survival and limit ischaemia/reperfusion injury ${ }^{36}$; determining if these systems are also active in the placenta requires further investigation. Additionally, the human placenta has distinct functional cell types-cytotrophoblasts and the syncytiotrophoblast-that possess mitochondria with very different structures and functions ${ }^{20}$. Further examination is required into the regulation of mitochondrial-related processes such as fission/fusion and apoptosis in cytotrophoblasts and the syncytiotrophoblast, and how these processes may differ in these important cell types.

\section{Acknowledgements}

We thank the staff and patients of the Gold Coast University Hospital, Australia, and the Royal Brisbane and Women's Hospital, Australia, for providing samples, and Georgia McEnro, Anna Alvsaker, Lauren Wendt (Griffith University), and Nykola L Kent for technical support. This work was supported by grants-in-aid from the National Health and Medical Research Council (grant \# APP569693), and the Gold Coast Hospital Foundation.

\section{Author details}

'School of Medical Science, Griffith University, Gold Coast Campus, Southport, QLD, Australia. ${ }^{2}$ School of Biomedical Sciences, The University of Queensland, St Lucia, QLD, Australia. ${ }^{3}$ Royal Brisbane and Women's Hospital, University of Queensland Centre for Clinical Research, Herston, QLD, Australia. ${ }^{4}$ School of Chemistry and Molecular Biosciences, The University of Queensland, St Lucia, QLD, Australia. ${ }^{5}$ Women and Newborns, Royal Brisbane and Women's Hospital, Herston, QLD 4101, Australia

\section{Compliance with ethical standards}

\section{Conflict of interest}

The authors declare that they have no conflict of interest.

\section{Publisher's note}

Springer Nature remains neutral with regard to jurisdictional claims in published maps and institutional affiliations.

Supplementary Information accompanies this paper at (https://doi.org/ 10.1038/s41419-018-1190-9).

Received: 15 June 2018 Accepted: 22 October 2018

Published online: 19 November 2018

\section{References}

1. Hutcheon, J. A., Lisonkova, S. \& Joseph, K. S. Epidemiology of pre-eclampsia and the other hypertensive disorders of pregnancy. Best practice \& research. Clin. Obstet. Gynaecol. 25, 391-403 (2011).

2. von Dadelszen, P. \& Magee, L. A. Pre-eclampsia: an update. Curr. Hypertens. Rep. 16, 454 (2014).

3. Liu, L. et al. Global, regional, and national causes of under-5 mortality in 200015: an updated systematic analysis with implications for the Sustainable Development Goals. Lancet 388, 3027-3035 (2016).

4. Leavey, K, Bainbridge, S. A. \& Cox, B. J. Large scale aggregate microarray analysis reveals three distinct molecular subclasses of human preeclampsia. PLOS ONE 10, e0116508 (2015).

5. American College of Obstetricians and Gynecologists. Hypertension in pregnancy. Report of the American College of Obstetricians and Gynecologists' Task Force on Hypertension in Pregnancy. Obstet Gynecol. 122, 1122-31. https://doi.org/10.1097/01.AOG.0000437382.03963.88. (2013).
6. Redman, C. W. G. \& Sargent, I. L. Placental debris, qxidative stress and preeclampsia. Placenta 21, 597-602 (2000).

7. Redman, C. W. \& Staff, A. C. Preeclampsia, biomarkers, syncytiotrophoblast stress, and placental capacity. Am. J. Obstet. Gynecol. 213, S9.e1 (2015). S9-11.

8. Mailloux, R. J. Teaching the fundamentals of electron transfer reactions in mitochondria and the production and detection of reactive oxygen species. Redox Biol. 4, 381-398 (2015).

9. Youle, R. J. \& van der Bliek, A. M. Mitochondrial fission, fusion, and stress. Science 337, 1062-1065 (2012).

10. Marengo, B. et al. Redox Homeostasis and Cellular Antioxidant Systems: Crucial Players in Cancer Growth and Therapy. Oxid. Med. Cell Longev. 2016, 6235641 (2016).

11. Chistiakov, D. A., Shkurat, T. P., Melnichenko, A. A., Grechko, A. V. \& Orekhov, A. N. The role of mitochondrial dysfunction in cardiovascular disease: a brief review. Ann. Med. 50, 121-127 (2018).

12. Ong, S. B. \& Hausenloy, D. J. Mitochondrial Dynamics as a Therapeutic Target for Treating Cardiac Diseases. Handb. Exp. Pharmacol. 240, 251-279 (2017).

13. Neri, M., Riezzo, I., Pascale, N., Pomara, C. \& Turillazzi, E. Ischemia/Reperfusion Injury following Acute Myocardial Infarction: A Critical Issue for Clinicians and Forensic Pathologists. Mediat. Inflamm. 2017, 7018393 (2017).

14. Holloszy, J. O. Biochemical adaptations in muscle. Effects of exercise on mitochondrial oxygen uptake and respiratory enzyme activity in skeletal muscle. J. Biol. Chem. 242, 2278-2282 (1967).

15. Broskey, N. T. et al. Skeletal muscle mitochondria in the elderly: effects of physical fitness and exercise training. J. Clin. Endocrinol. Metab. 99, 1852-1861 (2014).

16. Heusch, G., Botker, H. E., Przyklenk, K., Redington, A. \& Yellon, D. Remote ischemic conditioning. J. Am. Coll. Cardiol. 65, 177-195 (2015).

17. Murry, C. E., Jennings, R. B. \& Reimer, K. A. Preconditioning with ischemia: a delay of lethal cell injury in ischemic myocardium. Circulation 74, 1124-1136 (1986).

18. Cadenas, S. ROS and redox signaling in myocardial ischemia-reperfusion injury and cardioprotection. Free Radic. Biol. Med. 117, 76-89 (2018).

19. Vanderlelie, J. et al. Increased biological oxidation and reduced anti-oxidant enzyme activity in pre-eclamptic placentae. Placenta 26, 53-58 (2005).

20. Holland, O. et al. Review: Placental mitochondrial function and structure in gestational disorders. Placenta 54, 2-9 (2016).

21. Khera, A. et al. Selenium supplementation induces mitochondrial biogenesis in trophoblasts. Placenta 36, 863-869 (2015).

22. Khera, A., Vanderlelie, J. J., Holland, O. \& Perkins, A. V. Overexpression of Endogenous Anti-Oxidants with Selenium Supplementation Protects Trophoblast Cells from Reactive Oxygen Species-Induced Apoptosis in a BCl-2Dependent Manner. Biol. Trace Elem. Res. 177, 394-403 (2016).

23. Khera, A., Vanderlelie, J. J. \& Perkins, A. V. Selenium supplementation protects trophoblast cells from mitochondrial oxidative stress. Placenta 34, 594-598 (2013).

24. Rumbold, A., Duley, L., Crowther, C. A. \& Haslam, R. R. Antioxidants for preventing pre-eclampsia. Cochrane Database Syst. Rev. 23, Cd004227 (2008).

25. Salles, A. M., Galvao, T. F., Silva, M. T., Motta, L. C. \& Pereira, M. G. Antioxidants for preventing preeclampsia: a systematic review. ScientificWorldJournal 2012 243476 (2012).

26. Nezu, M. et al. Nrf2 inactivation enhances placental angiogenesis in a preeclampsia mouse model and improves maternal and fetal outcomes. Sci. Signal. 10, https://doi.org/10.1126/scisignal.aam5711 (2017).

27. Holland, O. J. et al. Changes in mitochondrial respiration in the human placenta over gestation. Placenta 57, 102-112 (2017).

28. Bustamante, J. et al. Oxygen metabolism in human placenta mitochondria. J. Bioenerg. Biomembr. 46, 459-469 (2014).

29. Olivera, A. A. \& Meigs, R. A. Mitochondria from human term placenta I. Isolation and assay conditions for oxidative phosphonylation. Biochim. Biophys. Acta 376, 426-435 (1975).

30. Fontana-Ayoub, M., Fasching, M. \& Gnaiger, E. Selected media and chemicals fro respirometry with mitochondrial preperations. Mitochondr Physiol. Netw. 17, 1-9 (2014).

31. Re, R. et al. Antioxidant activity applying an improved ABTS radical cation decolorization assay. Free Radic. Biol. Med. 26, 1231-1237 (1999).

32. Erel, O. A novel automated direct measurement method for total antioxidant capacity using a new generation, more stable ABTS radical cation. Clin. Biochem. 37, 277-285 (2004). 
33. Smith, A. D. \& Levander, O. A. High-throughput 96-well microplate assays for determining specific activities of glutathione peroxidase and thioredoxin reductase. Methods Enzymol. 347, 113-121 (2002).

34. Eigentler, A. et al. Laboratory protocol: Citrate synthase. Mitochondrial marker enzyme. Mitochondr Physiol. Netw. 17, 1-11 (2015).

35. Pham, T., Loiselle, D., Power, A. \& Hickey, A. J. Mitochondrial inefficiencies and anoxic ATP hydrolysis capacities in diabetic rat heart. Am. J. Physiol. Cell Physiol. 307, C499-C507 (2014)

36. Kalogeris, T., Bao, Y. \& Korthuis, R. J. Mitochondrial reactive oxygen species: a double edged sword in ischemia/reperfusion vs preconditioning. Redox Biol. 2, 702-714 (2014)

37. Powe, C. E., Levine, R. J. \& Karumanchi, S. A. Preeclampsia, a disease of the maternal endothelium: the role of antiangiogenic factors and implications for later cardiovascular disease. Circulation 123, 2856-2869 (2011).

38. Chamley, L. W. et al. Review: where is the maternofetal interface? Placenta $\mathbf{3 5}$ Suppl, S74-S80 (2014)

39. Cohen, J. M. et al. Maternal Antioxidant Levels in Pregnancy and Risk of Preeclampsia and Small for Gestational Age Birth: A Systematic Review and Meta-Analysis. PLoS One 10, e0135192 (2015).

40. Rahimi, Z. et al. Matrix metalloproteinase-2 C-735T and its interaction with matrix metalloproteinase-7 A-181G polymorphism are associated with the risk of preeclampsia: influence on total antioxidant capacity and blood pressure. J. Obstet. Gynaecol. 38, 327-332 (2018).

41. Can, M., Guven, B., Bektas, S. \& Arikan, I. Oxidative stress and apoptosis in preeclampsia. Tissue Cell 46, 477-481 (2014).

42. Aban, M. et al. Expression of nuclear factor-kappa B and placental apoptosis in pregnancies complicated with intrauterine growth restriction and preeclampsia: an immunohistochemical study. Tohoku J. Exp. Med. 204, 195-202 (2004).

43. Vaughan, J. E. \& Walsh, S. W. Activation of NF-kappaB in placentas of women with preeclampsia. Hypertens. Pregnancy 31, 243-251 (2012).

44. Harmon, A. C. et al. The role of inflammation in the pathology of preeclampsia. Clin. Sci. 130, 409-419 (2016).

45. Buhimschi, I. A. et al. Protein misfolding, congophilia, oligomerization, and defective amyloid processing in preeclampsia. Sci. Transl. Med. 6, 245ra292 (2014).

46. Zhao, S. et al. Proteases and sFlt-1 release in the human placenta. Placenta $\mathbf{3 1}$ 512-518 (2010).

47. Hu, T. et al. Increased ADAM10 expression in preeclamptic placentas is associated with decreased expression of hydrogen sulfide production enzymes. Placenta 36, 947-950 (2015)

48. Yang, $Y$. et al. Self-control of HGF regulation on human trophoblast cell invasion via enhancing c-Met receptor shedding by ADAM10 and ADAM17. J. Clin. Endocrinol. Metab. 97, E1390-E1401 (2012).

49. Clifton, V. L., Vanderlelie, J. \& Perkins, A. V. Increased anti-oxidant enzyme activity and biological oxidation in placentae of pregnancies complicated by maternal asthma. Placenta 26, 773-779 (2005).

50. Bereiter-Hahn, J. \& Voth, M. Dynamics of mitochondria in living cells: shape changes, dislocations, fusion, and fission of mitochondria. Microsc. Res. Tech. 27, 198-219 (1994).

51. Faccenda, D. et al. Control of Mitochondrial Remodeling by the ATPase Inhibitory Factor 1 Unveils a Pro-survival Relay via OPA1. Cell Rep. 18, 1869-1883 (2017).
52. Vishnyakova, P. A. et al. Mitochondrial role in adaptive response to stress conditions in preeclampsia. Sci. Rep. 6, 32410 (2016).

53. Ausman, J. et al. Ceramide-induced BOK promotes mitochondrial fission in preeclampsia. Cell Death Dis. 9, 298 (2018).

54. Allaire, A. D., Ballenger, K. A., Wells, S. R., McMahon, M. J. \& Lessey, B. A. Placental apoptosis in preeclampsia. Obstet. Gynecol. 96, 271-276 (2000).

55. DiFederico, E., Genbacev, O. \& Fisher, S. J. Preeclampsia is associated with widespread apoptosis of placental cytotrophoblasts within the uterine wall. Am. J. Pathol. 155, 293-301 (1999)

56. Leung, D. N., Smith, S. C., To, K. F., Sahota, D. S. \& Baker, P. N. Increased placental apoptosis in pregnancies complicated by preeclampsia. Am. J. Obstet. Gynecol. 184, 1249-1250 (2001).

57. Burton, G. J., Yung, H. W., Cindrova-Davies, T. \& Charnock-Jones, D. S. Placental endoplasmic reticulum stress and oxidative stress in the pathophysiology of unexplained intrauterine growth restriction and early onset preeclampsia. Placenta 30 Suppl A, S43-S48 (2009).

58. Momoi, T. Caspases involved in ER stress-mediated cell death. J. Chem Neuroanat. 28, 101-105 (2004).

59. Burton, G. J., Yung, H. W. \& Murray, A. J. Mitochondrial - Endoplasmic reticulum interactions in the trophoblast: Stress and senescence. Placenta 52, 146-155 (2016).

60. Huppertz, B., Kadyrov, M. \& Kingdom, J. C. Apoptosis and its role in the trophoblast. Am. J. Obstet. Gynecol. 195, 29-39 (2006).

61. Greggio, C. et al. Enhanced Respiratory Chain Supercomplex Formation in Response to Exercise in Human Skeletal Muscle. Cell. Metab. 25, 301-311 (2017).

62. Lopez-Fabuel, I. et al. Complex I assembly into supercomplexes determines differential mitochondrial ROS production in neurons and astrocytes. Proc. Natl Acad. Sci. USA 113, 13063-13068 (2016).

63. Maranzana, E., Barbero, G., Falasca, A. I., Lenaz, G. \& Genova, M. L. Mitochondrial respiratory supercomplex association limits production of reactive oxygen species from complex I. Antioxid. Redox Signal. 19, 1469-1480 (2013).

64. Pesta, D. et al. Similar qualitative and quantitative changes of mitochondria respiration following strength and endurance training in normoxia and hypoxia in sedentary humans. American journal of physiology. Regul. Integr. Comp. Physiol. 301, R1078-R1087 (2011).

65. Cindrova-Davies, T. et al. Oxidative stress, gene expression, and protein changes induced in the human placenta during labor. Am. J. Pathol. 171 1168-1179 (2007).

66. Diamant, S., Kissilevitz, R. \& Diamant, Y. Lipid peroxidation system in human placental tissue: general properties and the influence of gestational age. Biol. Reprod. 23, 776-781 (1980).

67. Many, A. \& Roberts, J. M. Increased xanthine oxidase during labour-implications for oxidative stress. Placenta 18, 725-726 (1997).

68. Lee, K. J. et al. Global gene expression changes induced in the human placenta during labor. Placenta 31, 698-704 (2010).

69. Truksa, J. et al. Mitochondrially targeted vitamin E succinate modulates expression of mitochondrial DNA transcripts and mitochondrial biogenesis. Antioxid. Redox Signal. 22, 883-900 (2015).

70. Venegas, V., Wang, J., Dimmock, D. \& Wong, L. J. Real-time quantitative PCR analysis of mitochondrial DNA content. Curr. Protoc. Human Genet. Chapter 19, Unit19.17 (2011). 